\title{
Enhanced Gain in Photonic Crystal Amplifiers
}

Ek, Sara; Semenova, Elizaveta; Hansen, Per Lunnemann; Yvind, Kresten; Mørk, Jesper

Published in:

Proceedings of International Conference on Transparent Optical Networks (ICTON)

Link to article, DOI:

10.1109/ICTON.2012.6253790

Publication date:

2012

Link back to DTU Orbit

Citation (APA):

Ek, S., Semenova, E., Hansen, P. L., Yvind, K., \& Mørk, J. (2012). Enhanced Gain in Photonic Crystal Amplifiers. In Proceedings of International Conference on Transparent Optical Networks (ICTON) https://doi.org/10.1109/ICTON.2012.6253790

\section{General rights}

Copyright and moral rights for the publications made accessible in the public portal are retained by the authors and/or other copyright owners and it is a condition of accessing publications that users recognise and abide by the legal requirements associated with these rights.

- Users may download and print one copy of any publication from the public portal for the purpose of private study or research.

- You may not further distribute the material or use it for any profit-making activity or commercial gain

- You may freely distribute the URL identifying the publication in the public portal

If you believe that this document breaches copyright please contact us providing details, and we will remove access to the work immediately and investigate your claim 


\title{
Enhanced Gain in Photonic Crystal Amplifiers
}

\author{
Sara Ek, Elizaveta Semenova, Per Lunnemann, Kresten Yvind, Jesper Mørk \\ DTU-Fotonik - Department of Photonic Engineering \\ Denmark Technical University, building 345, DK2800, Kongens Lyngby, Denmark \\ Tel: (+45) 4525 6354, Fax: (+45) 4593 6581, e-mail: saek@fotonik.dtu.dk
}

\begin{abstract}
We experimentally demonstrate enhanced gain in the slow-light regime of quantum well photonic crystal amplifiers. A strong gain enhancement is observed with the increase of the group refractive index, due to light slow-down. The slow light enhancement is shown in a amplified spontaneous emission study of a $1 \mathrm{QW}$ photonic crystal amplifier. Net gain is achieved which enables laser oscillation in photonic crystal micro cavities. The ability to freely tailor the dispersion in a semiconductor optical amplifier makes it possible to raise the optical gain considerably over a certain bandwidth. These results are promising for short and efficient semiconductor optical amplifiers. This effect will also benefit other devices, such as mode locked lasers.
\end{abstract}

Keywords: photonic crystal, semiconductor optical amplifier, amplified spontaneous emission, laser.

\section{INTRODUCTION}

Slow light has been an active topic of research for the last decade, both due to fundamental interest in understanding light-matter interactions as well exploiting these effects for improving the performance of photonic devices or realizing new functionalities. Photonic crystal $(\mathrm{PhC})$ line defect waveguides constitute an interesting platform for exploiting slow light effects and much work has been performed on passive waveguide structures [1]-[5].

When including layers of QWs or QDs in the PhC membrane slab, one can control both the optical and electronic properties in an active $\mathrm{PhC}$ waveguide. By exploiting the slow light effect in active $\mathrm{PhC}$ waveguides, it has been suggested that an efficient, ultra compact semiconductor optical amplifier (SOA) can be achieved [6]. The device length can be drastically decreased compared to conventional ridge SOAs due to slow light enhanced light-matter interaction [7]. Such a device is desired for compact photonic chips and interconnects, e.g. for chipto-chip or board-to-board links. Moreover, optical amplification is essential on a photonic integrated chip. By compensating for the attenuation more functionalities can be included. $\mathrm{PhC}$ amplifiers have proven challenging to realize experimentally though, and to the best of our knowledge there are no experimental demonstrations of gain in broad band $\mathrm{PhC}$ amplifiers. There are only few contributions in the literature on the study of the amplified SE from active PhC devices; in reference [8] investigations were carried out on highly multimoded waveguides with three rows of missing holes, not suitable for high-speed operation, and Raineri et al. presents optical amplification within a resonance whose quality factor is Q 1200 [9]. In this work enhanced net gain in $\mathrm{PhC}$ amplifiers is demonstrated, the enhancement is shown to correlate well with the increase of the group refractive index due to light slow down. The devices have nearly zero input and output reflection coefficients and we emphasize that we focus on the output light intensity rather than the modification of the spontaneous emission decay time originating from the Purcell effect [10].

The slow light enhanced gain was also investigated in PhC laser structures. There are many demonstrations of lasing in photonic crystal structures [11]-[13], but most of them rely on the realization of a high-Q cavity in order to achieve lasing at a small net gain. There are only a few contributions in the literature, where lasing is explained by gain enhancement at low group velocities [14]-[16] Here we present lasing in PhC micro cavities which verifies that net gain is achieved in the $\mathrm{PhC}$ waveguides.

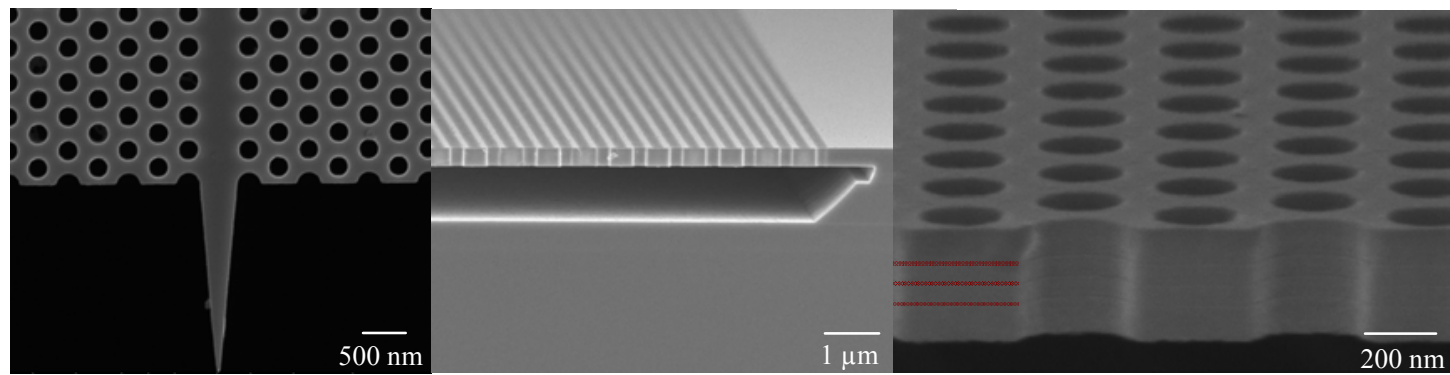

Fig. 1. SEM images of the fabricated devices. a) top view of the photonic crystal waveguide with taper b) a cross section view of the slab. There is a $1 \mu \mathrm{m}$ thick air-gap under the $330 \mathrm{~nm}$ thick active membrane. c) Zoom-in showing three layers of QD incorporated in the slab. The red lines are there for guiding the eye. 


\section{DESIGN AND FABRICATION}

The wafers were epitaxially grown in a metal-organic vapour phase epitaxi chamber (MOVPE). A $200 \mathrm{~nm}$ layer of $\mathrm{Si}_{3} \mathrm{~N}_{4}$ was deposited followed by a $500 \mathrm{~nm}$ thick layer of a positive e-beam resist (zep520A). The patterning was done using an e-beam writer (JEOL-JBX9300FS). The pattern was transferred to the $\mathrm{Si}_{3} \mathrm{~N}_{4}$ by $\mathrm{CHF}_{3} / \mathrm{O}_{2} \mathrm{RIE}$ and further transferred to the semiconductor by cyclic $\mathrm{CH}_{4} / \mathrm{H}_{2}-\mathrm{O}_{2}$ RIE after resist removal. Beneath the active slab there is a $1 \mu \mathrm{m}$ thick sacrificial layer, which is etched away using $\mathrm{HCl}$ selective wet-etching in order to obtain an air -slab structure. The period of the triangular PC is $380 \mathrm{~nm}$, the airhole diameter $200 \mathrm{~nm}$ and the waveguide is a single row of missing holes (defect waveguide). Scanning electron microscope (SEM) images of the fabricated device can be seen in Fig. 1. The coupling efficiency into the slab PC waveguide can be improved using an inverted taper, as demonstrated for a passive device by Tran et al. [17]. The taper is designed to improve mode matching, and to reduce the reflection on the end-facets.

\section{AMPLIFIED SPONTANEOUS EMISSION}

The spontaneous emission was measured using a lensed fibre aligned to the output taper of the device. The structure was optically pumped by a $980 \mathrm{~nm}$ laser diode, whose light was focused onto the sample from above using a cylindrical lens. This provides the energy enough to pump carriers up in the barriers of the QW which has its central emission wavelength centred at $1530 \mathrm{~nm}$. Up to $125 \mathrm{~mW}$ was focused onto the full length of the waveguide and its surrounding crystal. The emitted light was analysed in a liquid nitrogen cooled InGaAs spectrograph (Acton SP2500).

A measurement of the amplified spontaneous emission (ASE) as a function of pump power is presented in Fig. 2, aiming to compare the emission from the $\mathrm{PhC}$ waveguide with that from the wafer itself. Both the ASE from the $\mathrm{PhC}$ waveguide, with its band edge at $1558 \mathrm{~nm}$, and ASE from the wafer are measured at pump powers ranging from 7-125 mW, CW. When measuring the emitted light from the wafer itself, the fibre is aligned to be in level with the active layer, and focus is adjusted (distance between fibre and wafer) to optimize in-coupling into the fibre. The pump spot is a narrow stripe, identical to the one used for pumping the PhC waveguide, and light is detected at the cleaved facet. There is no defined waveguide in the bulk wafer, but a weak guiding is expected along the pump stripe, which is why the emission from the wafer also is denoted ASE.

Looking closer at the measurements in Fig. 2 b), heating is seen as a red-shift of the spectral features (about 2 $\mathrm{nm}$ ) between the low and high pump powers. For the bulk wafer (a) heating is not as big of an issue, because heat can dissipate down into the wafer, and does not accumulate in the pumped region as it does in the air-slab. Heating of the membrane causes carrier loss and therefore reduced output intensity. Studying the ASE $E_{P h C}$ normalized with the $\mathrm{ASE}_{\text {wafer }}$ displayed in c), an amplification of 2-4 times can be seen over the full spectra, with the maximum 4-fold enhancement in the slow light region close to the band edge. The spectral shape shows a clear signature of the photonic crystal dispersion.
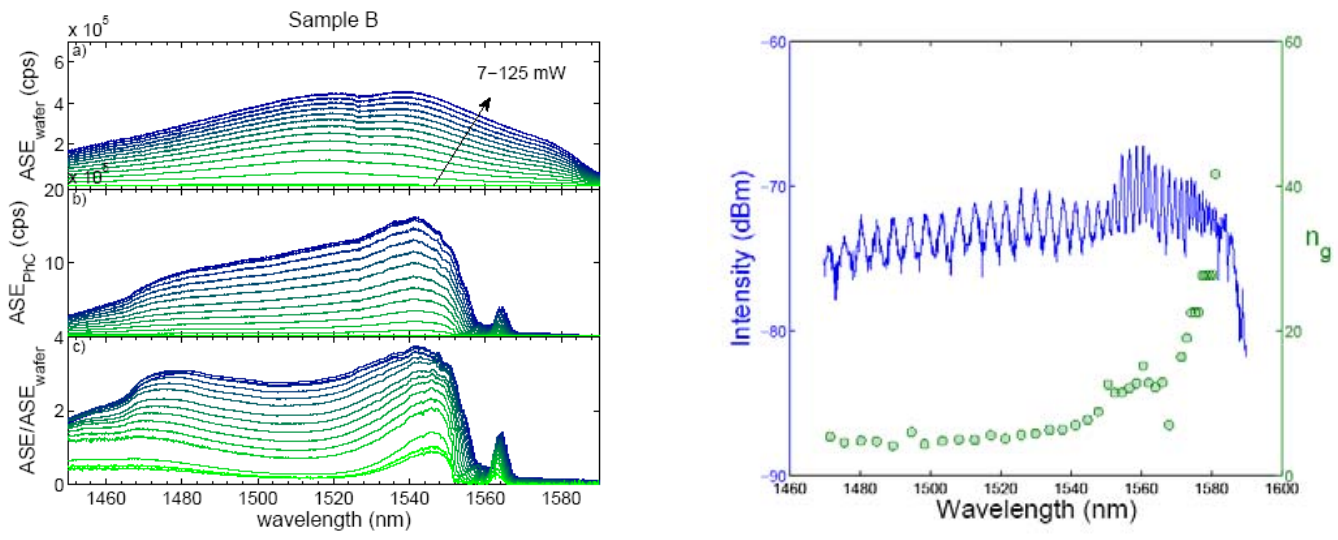

Fig. 3. Left: a) ASE spectra from $1 \mathrm{QW}$ wafer at CW pump powers ranging from 7-125 $\mathrm{mW}$ (980 nm). b) ASE spectra from a PhC waveguide on the same wafer. Pitch $380 \mathrm{~nm}$ and hole diameter $200 \mathrm{~nm}$. c) The normalized ASE, showing the enhancement in the slow light regime. (cps=counts per second) Right: Spontaneous emission from a $50 \mu \mathrm{m}$ short PhC waveguide with a pitch of $400 \mathrm{~nm}$ and a hole diameter of $210 \mathrm{~nm}$ and flat end facets $(\mathrm{RBW}=0.1 \mathrm{~nm})$. The deducted group index is shown in green

We measured the dispersion using a short $(50 \mu \mathrm{m}) \mathrm{PhC}$ waveguide with a pitch of $400 \mathrm{~nm}$ but without tapers. The flat end facets promote Fabry-Pérot oscillations from which the group velocity may be derived, and the shorter PC ensures better resolved peaks on the OSA. The emission from the short PC waveguide and the calculated group index is shown in Fig. 2 (right). Using the wavelength spacing $\Delta \lambda$ between adjacent FabryPérot peaks and the length $L$, the group index was calculated as $n_{g}=\lambda^{2} /(2 L \Delta \lambda)$. The decrease in the group velocity is significant close to the bandedge, where the groupvelocities on the order of $\mathrm{v}_{\mathrm{g}}=\mathrm{c} / \mathrm{n}_{\mathrm{g}}=\mathrm{c} / 40$ were achieved, which is a reasonable range to limit the influence of disorder effects [1]. 


\section{PHOTONIC CRYSTAL MICROLASER}

Laser cavities were also fabricated and characterized. All laser structures are 3 QD PhC structures in $340 \mathrm{~nm}$ thick membranes. Two laser designs are presented which differ in $\mathrm{PhC}$ design and output mirror configuration. The layout is similar to the amplifier design incorporating a $70 \mu \mathrm{m}$ long W1 micro cavity. A lattice constant of $400 \mathrm{~nm}$ is used with a variation of hole size to shift the lasing wavelength of the band edge laser. The line defect which forms the cavity extends $70 \mu \mathrm{m}$ into the $\mathrm{PhC}$ from the out-coupling mirror, where it is blocked by holes. The output mirror is made to be only partly reflective by inserting four holes $5 \mu \mathrm{m}$ away from the out-coupling taper. Calculations of the reflection and transmission spectra of the structures based on $2 \mathrm{D}$ finite difference time domain simulations, indicates that 4 holes results in $97 \%$ reflectivity for wavelengths within the photonic bandgap. A schematic over the laser configuration is shown to the right in Fig. 3. The lasers are optically pumped with $1 \mathrm{ps}$ long laser pulses at a wavelength of $800 \mathrm{~nm}$ and repetition rate of $270 \mathrm{kHz}$. All the tested devices show lasing operation at a wavelength corresponding to the band edge of each design, as seen in Fig. 3 (left). Laser emission spectra from three PhC cavities are shown, all with a hole diameter shift of $5 \mathrm{~nm}$ relative the previous. Such a shift in hole diameter is expected to move the bandedge about $8 \mathrm{~nm}$ towards shorter wavelengths, which corresponds to the experimentally observed wavelength shift.
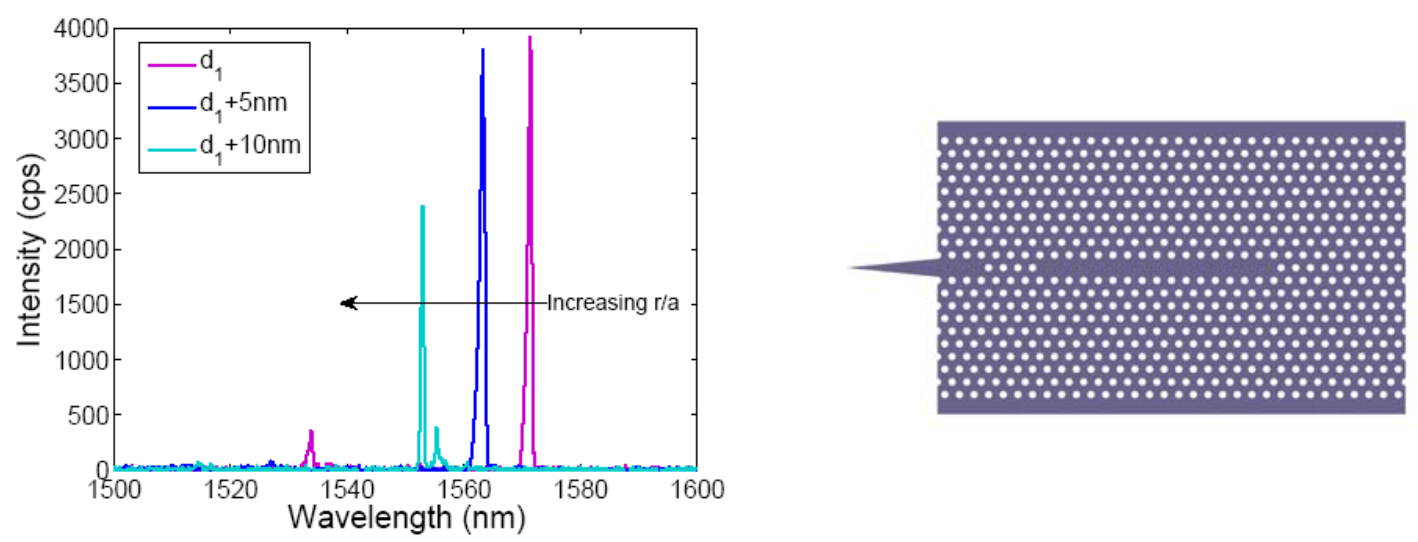

Fig. 3. Laser emission spectra from three $70 \mu \mathrm{m}$ long 3QD lasers with increasing hole size. The cavities are pumped with the output from a laser emitting $1 \mathrm{ps}$ long pulses at a wavelength of $800 \mathrm{~nm}$. All devices have a four-hole output mirror and a outcoupling taper as shown to the right.

The second laser design is a $50 \mu \mathrm{m}$ long cavity, which is a line defect terminated by $\mathrm{PhC}$ in one end and a cleaved facet in the other. The lattice constant of the $\mathrm{PhC}$ is $390 \mathrm{~nm}$ and the hole diameter in the fabricated laser is $132 \mathrm{~nm}$. The dispersion properties of the device are modified by altering the size of the holes in the first and second row closest to the waveguide; $r_{1}=95 \mathrm{~nm}$ and $r_{2}=156 \mathrm{~nm}$.

The same pumping scheme as for the first set of lasers is used and the power levels noted in the emission spectra, Fig. 4 (left), denotes the average pump power over the full pump spot which has an area of about $10 \mathrm{x}$ $400 \mu \mathrm{m}$. The emission is detected on the cooled InGaAs spectrograph, with a resolution of $0.15 \mathrm{~nm}$. Emission spectra at representative pump powers are shown in the figure. There are two spectral regions where laser oscillation occurs, around $1435 \mathrm{~nm}$ and at $1595 \mathrm{~nm}$. The integrated intensity as a function of average pump power is shown in Fig. 4 (right). The emission shows a threshold behavior at an average pump power of $0.3 \mathrm{~mW}$. A zoom in at low pump powers is seen in the inset.

A linewidth narrowing is observed at pump-powers up to $0.3 \mathrm{~mW}$, just above threshold. As the pump power increases the peak is broadened (compare emission at $0.31 \mathrm{~mW}$ and $1.4 \mathrm{~mW}$ ). Since the peak is not red shifted for higher pump powers, the broadening is not believed to be caused by heating. Rather, the large carrier density injected by the short, energetic pump pulses might introduce a deterministic chirp. The fact that nanocavity lasers are chirped under pulsed pumping is found in a recent report from Braive et al. [18].

\section{CONCLUSIONS}

We fabricated active photonic crystal slab defect waveguides, incorporating a single QW, and containing taper structures at input and output that lead to a small insertion loss and small residual reflection. Using these structures an enhancement of amplified spontaneous emission was observed that can be explained by the achievement of net gain in combination with light slow-down by the photonic crystal dispersion. PhC microcavities containing three layers of QDs were also fabricated and characterized. The demonstration of laser oscillation confirms that net gain is achieved in the $3 \mathrm{QD}$ photonic crystal devices. This platform is promising for realizing compact photonic crystal semiconductor optical amplifiers as well as mode-locked lasers. 

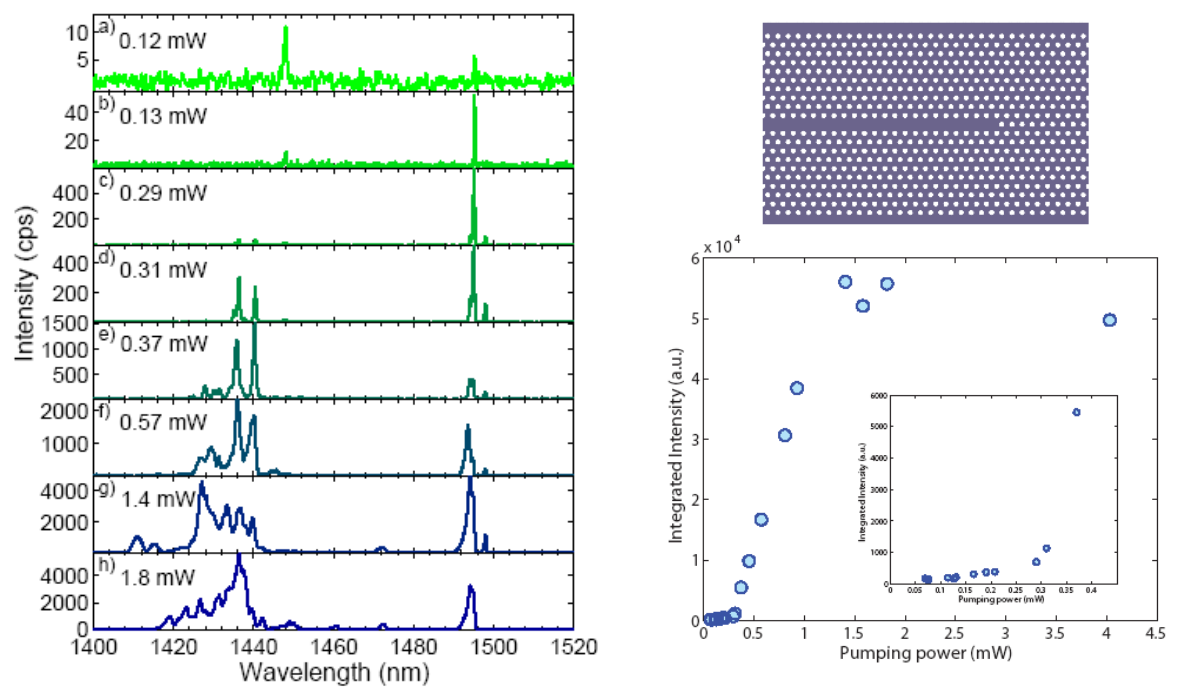

Fig. 2. Left: Laser emission spectra from a $50 \mu \mathrm{m}$ long 3 QD cavity. The indicated power levels are average powers of the pulsed pump laser emitting at $800 \mathrm{~nm}$. The holes closest to the waveguide are of different size than the rest of the lattice, leading to emission at two wavelength regions. Right: the top figure illustrates the cavity configuration. The cleaved facet serves as a partly reflective mirror. The bottom figure show the measured Pin/Pout curve. The inset is a zoom-in at low pump powers.

\section{ACKNOWLEDGEMENTS}

The authors thank A. De Rossi and S. Combrie at Thales Research and Technology. This work is funded by NATEC Centre of excellence.

\section{REFERENCES}

[1] T. F. Krauss, Slow light in photonic crystal waveguides, Journal of Physics D: Applied Physics, vol. 40, pp. 2666-2670, 2007.

[2] Y.A. Vlasov, et al.: Active control of slow light on a chip with photonic crystal waveguides, Nature, vol. 438, pp 65-69, 2005.

[3] M. Loncar, T. Doll, and J. Vuckovic, Design and fabrication of silicon photonic crystal optical waveguides, Journal of Lightwave Technology, vol. 18, pp. 1402-1411, 2000.

[4] L. Frandsen, et al.: Photonic crystal waveguides with semi-slow light and tailored dispersion properties, Optics Express, vol. 20, pp 9444-9450, 2006.

[5] M. Kotylar, et al.: Low-loss photonic crystal defect waveguides in InP, Applied Physics Letters, vol. 84, pp. 3588, 2004.

[6] E. Mizuta, H. Watanabe and T. Baba, All Semiconductor Low- $\Delta$ Photonic Crystal Waveguide for Semiconductor Optical Amplifier, Japanese Journal of Applied Physics, vol. 45, pp. 6116-6120, 2006.

[7] T. Baba, Slow light in photonic crystals, Nature Photonics, vol. 2, pp. 465-473, 2008.

[8] E. Schwoob, Enhanced gain measurement at mode singularities in InP-based photonic crystal waveguides, Optics Express, vol. 12, pp. 1569-1574, 2004.

[9] F. Raineri, Optical amplification in two-dimensional photonic crystals, Applied Physics Letters, vol. 86, pp. 091111, 2005.

[10] T. Lund-Hansen, et al.: Experimental realization of highly-efficient broadband coupling of single quantum dots to a photonic crystal waveguide, Physical Review Letters, vol. 101, pp. 113903, 2008.

[11] O. Painter, et al.: Two-Dimensional Photonic Band-Gap Defect Mode Laser, Science, vol. 11, pp. 1819$1821,1999$.

[12] B. Ellis, et al.: Ultralow-threshold electrically pumped quantum-dot photonic-crystal nanocavity laser, Nature Photonics, vol 5, pp. 297-300, 2011.

[13] K. Inoshita and T. Baba, Room-Temperature Lasing Characteristics of Bend and Branch in Photonic Crystal Waveguide, Japanese Journal of Applied Physics, vol. 42, pp. 6887-6891, 2003.

[14] X. Ming-Xin, et al.: Slow Light Effect and Multimode Lasing in a Photonic Crystal Waveguide Microlaser, Chinese Physics Letters, vol. 27, pp. 024213, 2010.

[15] K. Kiyota, et al.: Various low group velocity effects in photonic crystal line defect waveguides and their demonstration by laser oscillation, Applied Physics Letters, vol. 88, pp. 201904, 2006.

[16] K. Inoue, et al.: InAs quantum-dot laser utilizing GaAs photonic-crystal line-defect waveguide, Optics express, vol. 12, pp. 5502-5509, 2004.

[17] Q. V. Tran, et al.: Photonic crystal membrane waveguides with low insertion losses, Applied Physics Letters, vol. 95, pp. 061105, 2009.

[18] R. Braive, et al.: Transient chirp in high-speed photonic-crystal quantum-dot lasers with controlled spontaneous emission, vol. 34, pp. 554-556, 2009. 